To appear in JGR, 2002

\title{
Imaging radar observations and theory of type I and type II quasiperiodic echoes
}

\author{
D. L. Hysell ${ }^{1}$, M. Yamamoto, and S. Fukao \\ Radio Science Center for Space and Atmosphere, Kyoto University, Uji, Japan
}

\begin{abstract}
.
Midlatitude $E$ region plasma irregularities have been investigated using the Middle and Upper Atmosphere (MU) radar in Shigaraki, Japan, and the Clemson 30 $\mathrm{MHz}$ radar in South Carolina, USA. A new in-beam imaging technique has been incorporated in the data analysis. Radar images reveal that the coherent backscatter associated with quasiperiodic (QP) echoes mainly arrives from spatially localized, elongated scattering regions and that striations in radar range-time-intensity (RTI) maps are the signatures of the migrations of these regions through the sparselyfilled illuminated volume. The scattering regions in question appear to maintain altitude as they drift. Refraction and finite aspect angle sensitivity permit field aligned irregularities in the regions to be detected by radars over a broad range of zenith angles. Circulation observed within the scattering regions is consistent with simulations of polarized $E$ region plasma clouds described in a companion paper, as is the occasional appearance of type I echoes. Several long, continuous bands of scatterers are also evident in the radar images.
\end{abstract}

\section{Introduction}

In the companion paper (Hysell et al. [2002], henceforth referred to as paper 1), we investigated the electrodynamics of irregular, patchy sporadic $E$ layers and plasma clouds in the nighttime midlatitude $E$ region. Such layers have been observed directly at Arecibo [Miller and Smith, 1978; Smith and Miller, 1980] and inferred from radio scintillation measurements [Bowman, 1989; Maruyama, 1991], and the morphology of the related quasi-periodic scintillations (QPS) has been shown to match that of QP echoes closely [Maruyama et al., 2000]. The source of the patchiness is not known, although Larsen [2000] has argued that it may be driven by neutral shear instabilities. Larsen [2000] further argued that these patchy layers are the direct sources of quasiperiodic echoes. Our theoretical investigation showed that large polarization electric fields and Hall currents can arise spontaneously in elongated layers and that the layers themselves are unstable to kilometer-scale primary plasma waves. The ingredients necessary for forming small-scale ir-

\footnotetext{
${ }^{1}$ On leave from the Department of Physics and Astronomy, Clemson University, Clemson, South Carolina, USA
}

regularities within the clouds, either directly by Farley Buneman instabilities or indirectly by mode coupling and plasma turbulence driven by the primary waves, seem to be present. However, links between these theoretical findings and observations of coherent scatter from midlatitude irregularities and QP echoes in particular need to be established.

Numerous theories intended to account for the characteristics of midlatitude $E$ region coherent scatter have been advanced in recent years [Woodman et al., 1991; Tsunoda et al., 1994; Haldoupis et al., 1996; Shalimov et al., 1998; Tsunoda, 1998; Kagan and Kelley, 1998; Rosado-Roman et al., 1999; Kagan and Kelley, 2000; Maruyama et al., 2000]. Validating these theories with conventional radar techniques like range-time-intensity (RTI) analysis, spectral analysis, single baseline interferometry, and beam swinging alone may not be possible, however. These techniques do not always distinguish clearly between spatial and temporal variations within the illuminated volume and cannot resolve fine structure in the directions perpendicular to the radar beam. The existing empirical database for QP echoes is somewhat ambiguous in that regard, and multiple theories appear to be broadly consistent with it. 
This paper presents observations of plasma irregularities in the midlatitude $E$ region made with the MU and Clemson radars using a new in-beam radar imaging technique. The technique, introduced at Jicamarca by Kudeki and Sürücü [1991] and developed by Woodman [1997] and Hysell and Woodman [1997], utilizes multiple interferometry baselines to resolve spatial-temporal ambiguity and to discern fine structure within the radar illuminated volume. Images produced with the technique will favor an interpretation of QP echoes as localized, drifting scattering regions sparsely distributed throughout the radar illuminated volume. Striations in RTI plots reflect the motion of the scattering regions toward or away from the radar. Telltale signs of circulation within the scattering regions support the idea that they are associated with elongated, polarized $E$ region plasma clouds drifting with the background wind. The detection of type I echoes immediately above a very elongated scattering region also support this picture. We further argue that plasma irregularities within localized, horizontally drifting plasma clouds can remain visible to coherent scatter radars for long periods of time due in large part to refraction.

We begin with an overview of a series of experiments performed with the Clemson $30 \mathrm{MHz}$ radar and the MU VHF radar which were used to study QP echoes and operated in imaging mode in the summer of 2001. After briefly describing the principles of radar imaging, we present a series of images constructed from the radar data and evaluate their connection to the theory outlined in paper 1. Finally, we summarize our findings an suggest directions for future research.

\section{Experimental overview}

Midlatitude $E$ region plasma irregularities were studied with the Clemson $30 \mathrm{MHz}$ coherent scatter radar on approximately 50 evenings between May and August, 2001 and with the MU VHF radar on three successive evenings in August, 2001. Strong $E$ region echoes were detected at Clemson on about half of all evenings and at the MU radar on all three evenings of operation. These radars are situated at similar geographic latitudes but produced qualitatively different datasets due to differences in their geomagnetic latitude, frequency, sensitivity, and hours of operation. Information about the processes responsible for quasiperiodic and other forms of echoes can be deduced from a comparison of the datasets. Table 1. lists the experiment parameters. The main beams of the MU and Clemson radars were directed toward geographic and geomagnetic north, respectively. Experiments at both sites were supported locally by ionosondes.

Figure 1 shows a representative example of the MU radar
Table 1. Table of radar parameters for the QP echo experiments.

\begin{tabular}{lrr}
\hline \multicolumn{1}{c}{ Parameter } & MU radar & Clemson radar \\
\hline Latitude $(\mathrm{deg})$ & $34.9 \mathrm{~N}$ & $34.7 \mathrm{~N}$ \\
Longitude $(\mathrm{deg})$ & $136.1 \mathrm{E}$ & $82.8 \mathrm{~W}$ \\
Dip angle $(\mathrm{deg})$ & 51 & 66.5 \\
Frequency $(\mathrm{MHz})$ & 46.5 & 29.8 \\
Peak power $(\mathrm{kW})$ & 1000 & 8 \\
IPP $(\mathrm{ms})$ & 1.5 & 4 \\
Coherent int. & 4 & 1 \\
Baud length $(\mathrm{km})$ & 0.3 & 1.5 \\
Code length $(\mathrm{n})$ & 32 & 28 \\
Receivers & 4 & 4 \\
Baselines & 11 & 9 \\
$d_{x}(\lambda)$ & 3.04 & 1.25 \\
$d_{y}(\lambda)$ & 5.27 & 5 \\
HPFB $($ deg $)$ & 3.6 & 10 \\
\hline
\end{tabular}

data from August 9, 2001, plotted in range-time-intensity (RTI) format. The figure is dominated by two distinct groups of QP echo striations separated by a period of relative inactivity lasting about an hour. The range rates, range extents, slope signs, and pseudo periods of the striations are consistent with what has been documented by Yamamoto et al. [1991, 1992]. Echoes observed on August 7 were similar to those shown here except that the striations occurred in three distinct groups, separated again by hour-long periods of inactivity. In contrast, Figure 2 shows echoes received by the MU radar on August 8, 2001. These echoes differ morphologically from those in Figure 1, failing to resemble quasiperiodic striations and instead appearing from 2115 to 2320 LT as a long, unbroken succession of amorphous "blobs".

Figure 3, meanwhile, shows representative data from the Clemson $30 \mathrm{MHz}$ radar taken on June 11, 2001 (UT date). This figure shows four distinct groupings of echoes separated in time by about $50 \mathrm{~min}$. The first two of these exhibit the characteristics of QP echo striations with nearly uniform, negative range rates and with large range extents in a few cases. In the third and fourth groupings, however, the echoes appear more like blobs or elongated blobs with positive, negative, or indeterminate range rates. The term "quasiperiodic" applies equally poorly to these echoes and the echoes registered by the MU radar on August 8. Although the majority of echoes seen by the Clemson radar in the summers of 1998 and 1999 resembled quasiperiodic striations with predominately negative and positive range rates in the evening and early morning, respectively, most of the echoes observed in 


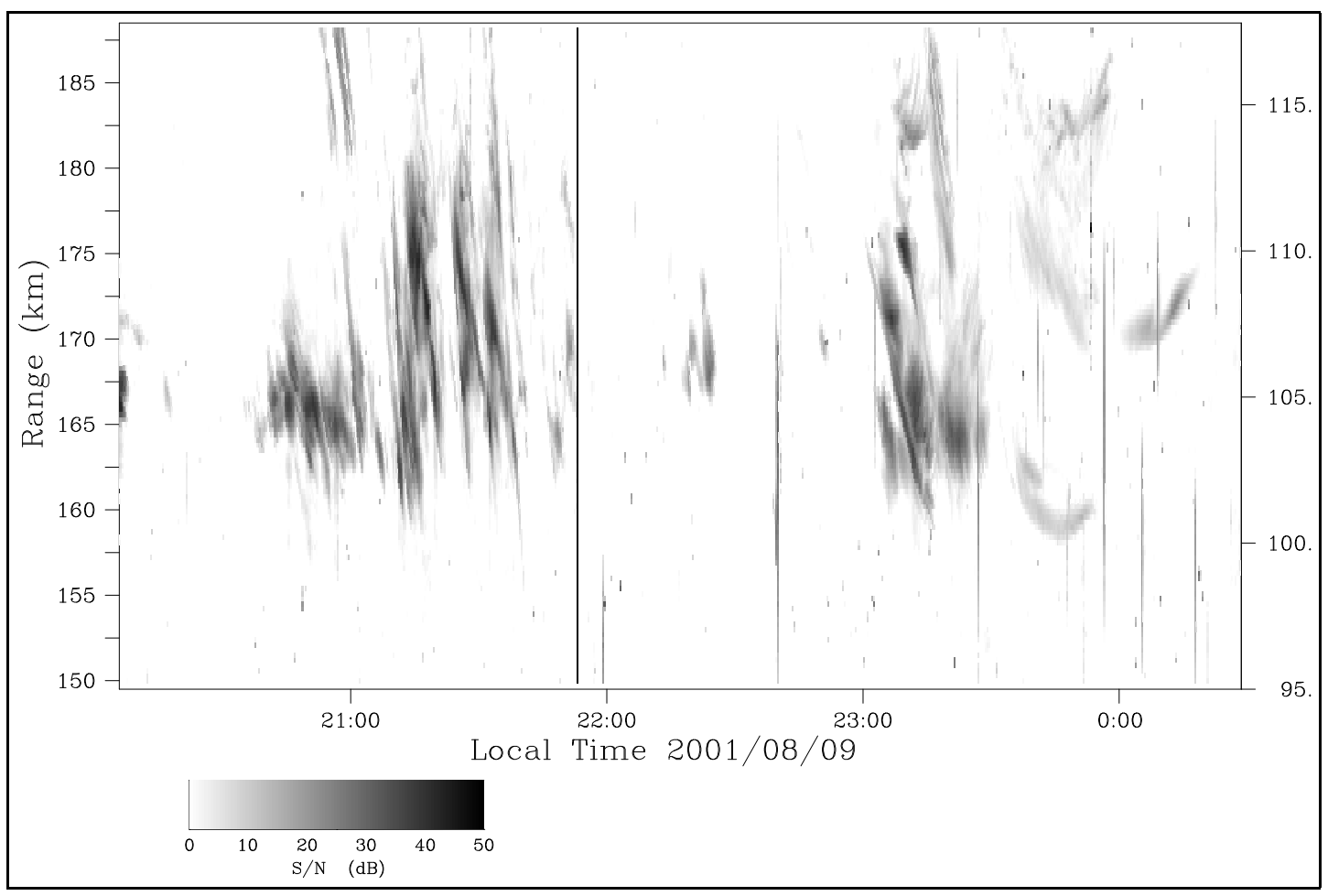

Figure 1. Range-time-intensity plot showing quasiperiodic echoes observed with the MU radar on August 9, 2001. Grayscales depict signal-to-noise ratios in $\mathrm{dB}$. A coarse approximation of the echo altitude in kilometers appear on the right axis of the plot (see text). 


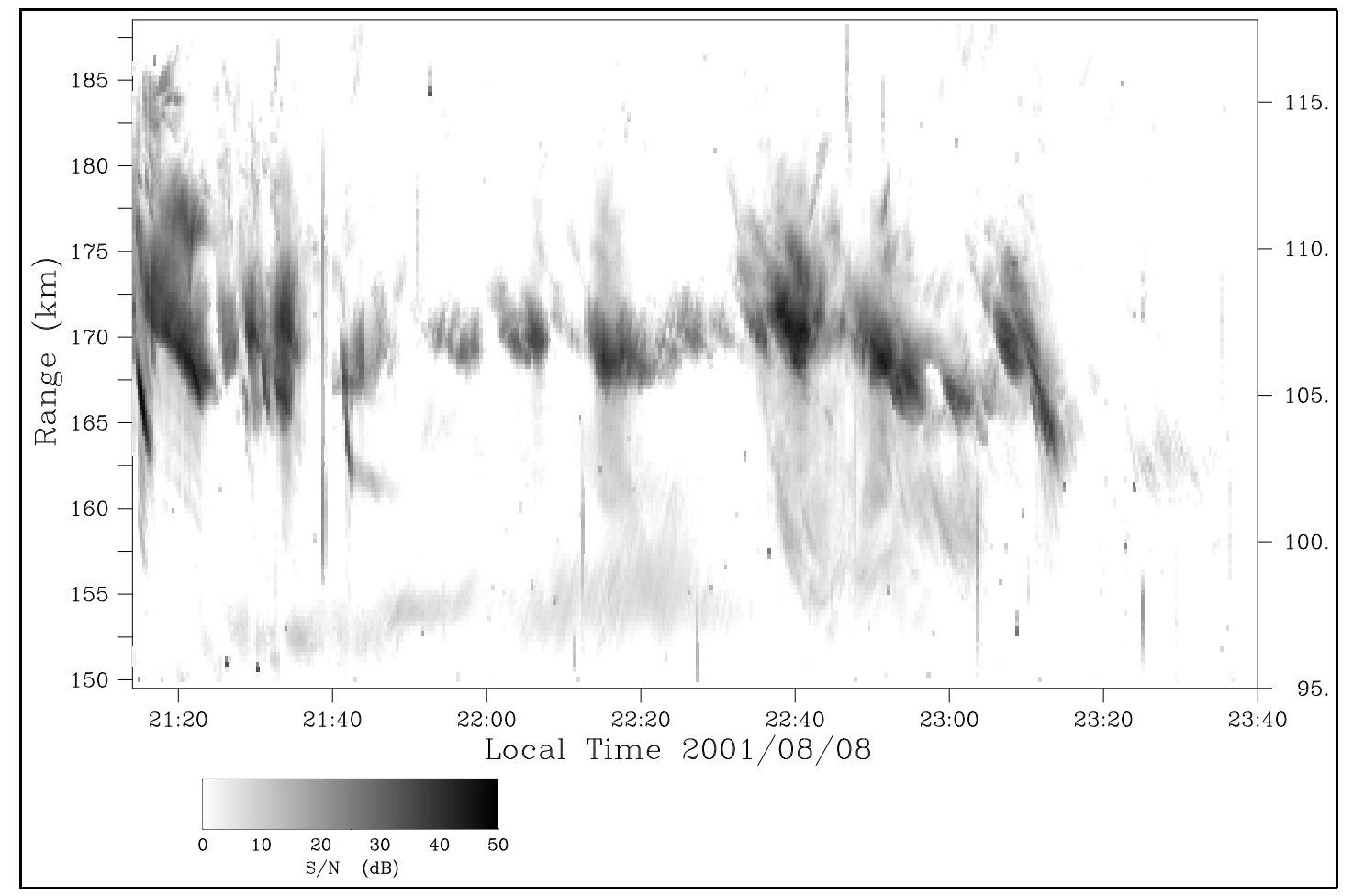

Figure 2. Range-time-intensity plot showing echoes observed with the MU radar on August 8,2001.

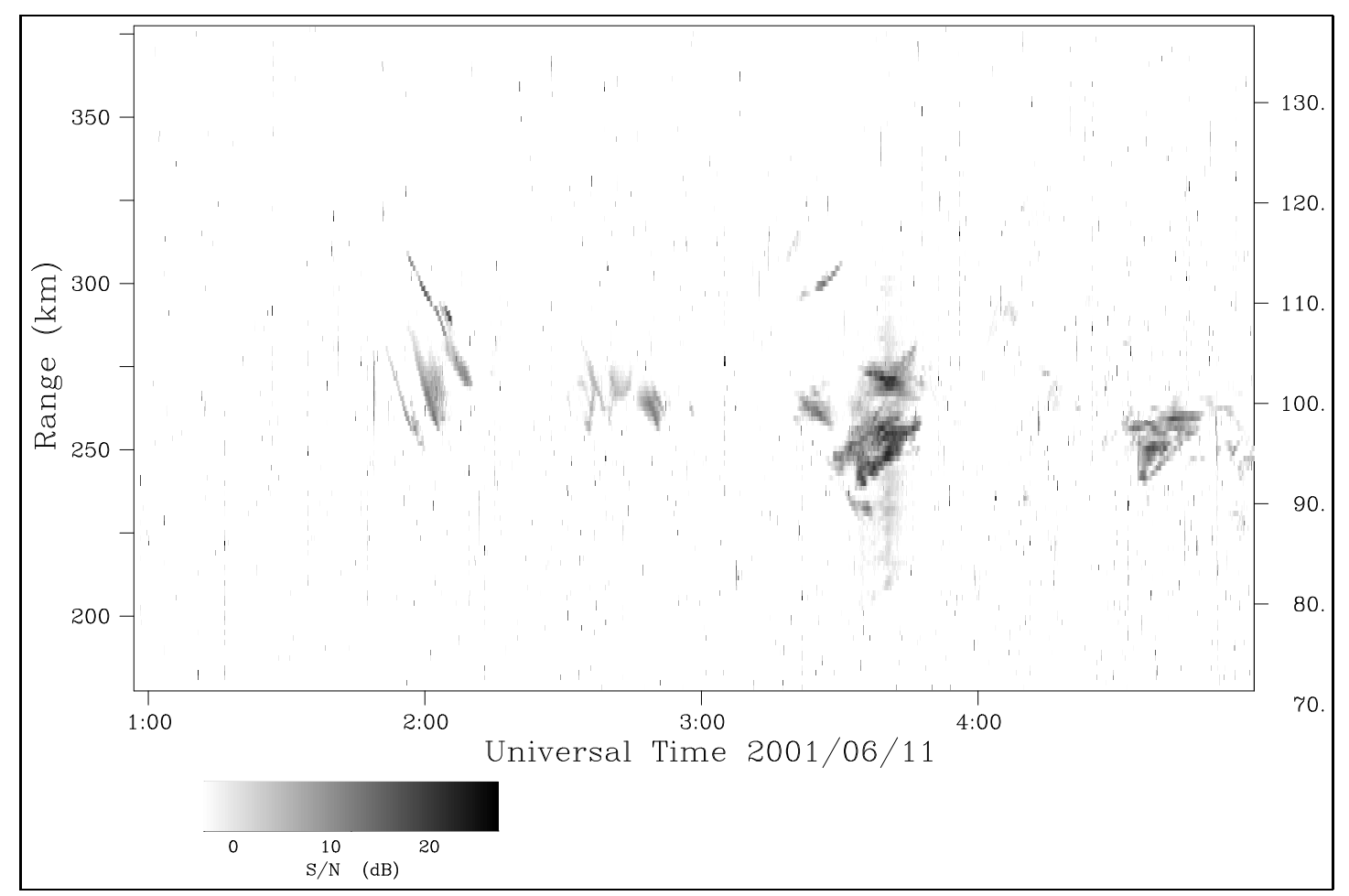

Figure 3. Range-time-intensity plot showing quasiperiodic echoes observed with the Clemson radar on June 11, 2001. Here, $\mathrm{UT}=\mathrm{LT}+5$ hours. 


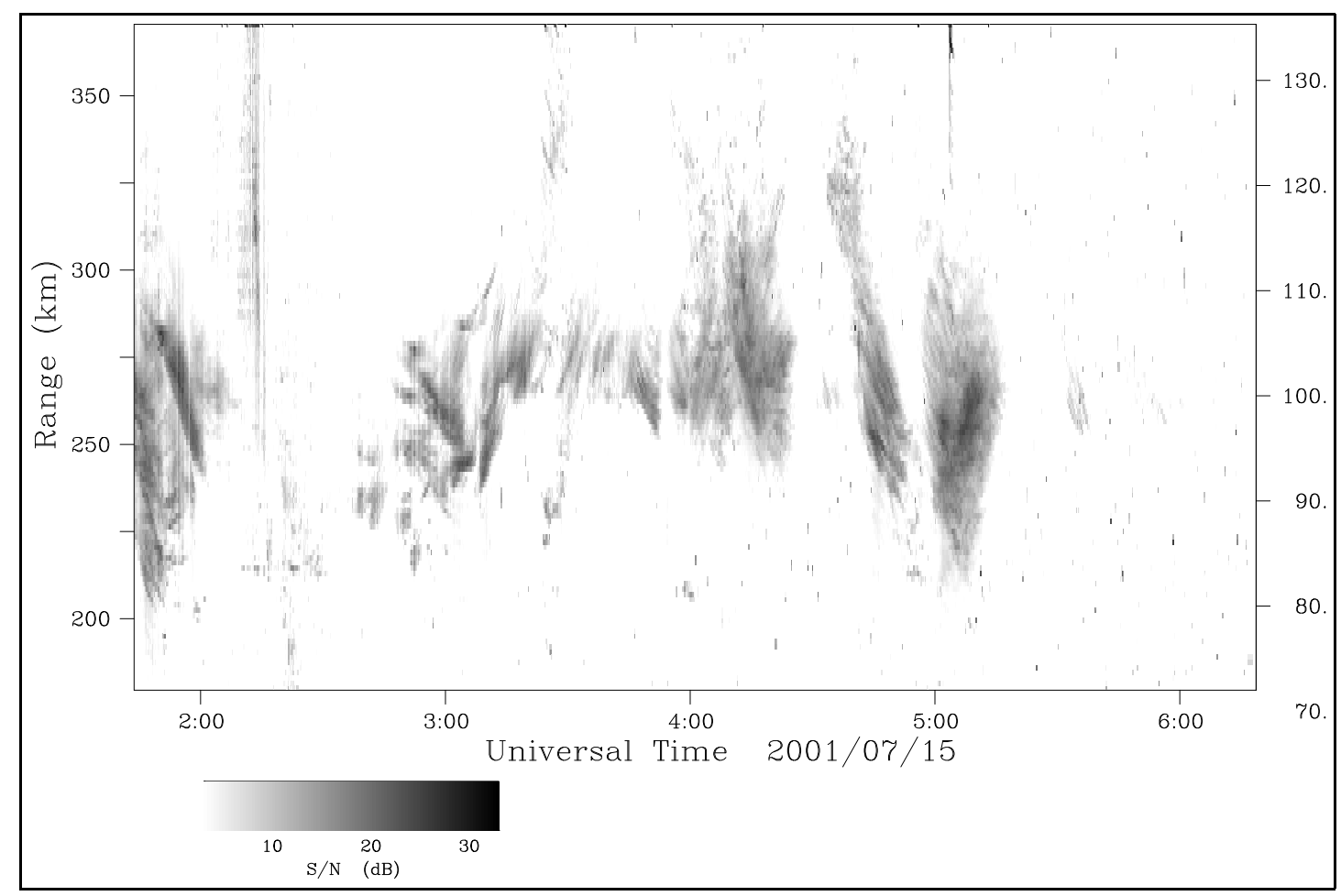

Figure 4. Range-time-intensity plot showing quasiperiodic echoes observed with the Clemson radar on June 15, 2001. Here, $\mathrm{UT}=\mathrm{LT}+5$ hours. Note that the echoes present at $0210 \mathrm{UT}$ in many range gates correspond to ground clutter.

2001 were blob-like [Hysell and Burcham, 2000]. Furthermore, those striated echoes seen in 2001 were equally likely to have positive and negative range rates. Figure 4 shows an example of particularly intense echoes received by the Clemson radar. QP echoes with both positive and negative range rates can be seen to overlap in this figure, ruling out the idea that they are somehow related to multiple, tilted, planar sporadic $E$ layers. Blob-like echoes are also present. It is noteworthy that the observations shown here, which include the only case of type I echoes received in 2001, were made during strong spread $F$ conditions.

The right axes of Figures 1-4 indicate the approximate altitudes from which the echoes could have arisen. The approximation in question is that radar signals propagate along straight lines through the azimuthal center of the radar beam at the elevation angle where field aligned irregularities exactly satisfy the condition for Bragg scatter. However, $\mathrm{Hy}$ sell and Burcham [2000] argued that these approximations are unreliable and that refraction, finite aspect angle sensitivity, and off-azimuth propagation make it impossible to assign a scattering altitude to an echo solely on the basis of range. The echoes that appear to have originated from altitudes as low as $90 \mathrm{~km}$ in Figure 3 and $80 \mathrm{~km}$ in Figure 4 actually originated at higher altitude but underwent signif- icant refraction. (Sporadic $E$ layers over Clemson had top frequencies of $6 \mathrm{MHz}$ at $0400 \mathrm{UT}$ on June 11 and $10 \mathrm{MHz}$ at various times on June 15, and top frequencies as high as 12 $\mathrm{MHz}$ were not uncommon during the evenings of the summer of 2001 there and at the MU radar.) Likewise, the long range echoes that might seem to have come from altitudes above about $120 \mathrm{~km}$ in Figure 4 actually came from lower altitudes but from large azimuth angles. In order to quantify the various effects that disassociate echo range and altitude, to study the configuration of the irregularities responsible for QP and other echoes, and to learn what the QP striations and blobs in RTI maps signify, we must turn to more incisive diagnostic tools like in-beam radar imaging.

True images of the backscatter "brightness" distribution (the distribution of backscatter intensity versus bearing) within the radar illuminated volume can be formed from interferometry data taken with multiple baselines. It is well known that interferometry with a single baseline yields two moments of the brightness distribution [Farley et al., 1981]. More baselines yield more moments, and a sufficient number of moments define an image in one or two dimensions. (Formally, the interferometry cross spectrum or "visibility" is related to the brightness distribution by an integral transform resembling a Fourier transform [Thompson, 1986].) Radar 


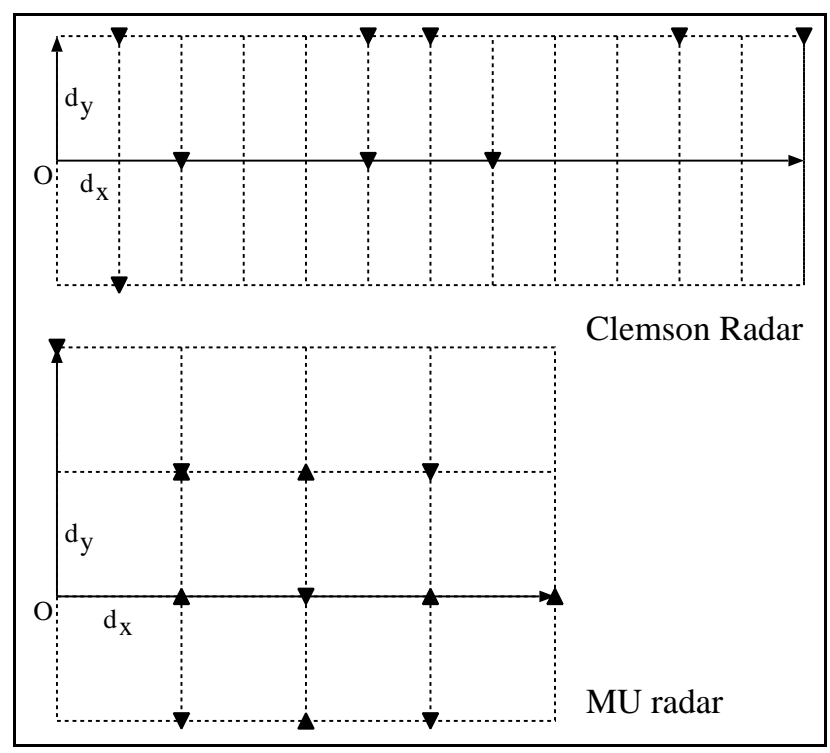

Figure 5. Configuration of interferometry baselines used with the MU and Clemson radars. Baseline lengths are integer multiples of the $d_{x}$ and $d_{y}$ factors listed in Table 1. Arrowhead directions indicate how the receivers were multiplexed.

range gating adds another spatial dimension. An important feature of in-beam radar imaging that distinguishes it from beam-swinging approaches is that the angular resolution of the technique is limited by the length of the longest interferometry baselines rather than the size of the main antenna array. The former can generally be increased economically whereas the latter generally cannot, and it is possible in practice to form very high resolution images with interferometry even using small radar systems like the Clemson radar. Images formed from different Doppler spectral components can be combined into composite images, conveying information about the spectral characteristics of different regions of the illuminated volume. Animated sequences of images reveal how the scatterers evolve over time as they travel through the illuminated volume.

Both the MU radar and the Clemson radar can use up to four receivers for interferometry. By receiving signals from four spatially separated antenna groups, cross-spectral measurements can be made on up to six nonredundant interferometry baselines. By multiplexing the four receivers to multiple set of antenna groups, this number can be increased. Cross spectra from 11 and 9 nonredundant, nonzero baselines were computed from the MU and Clemson radar data, respectively, in this way. The baseline configurations for both radars are illustrated in Figure 5. Here, the components of the baselines in the directions transverse to and parallel to the radar main beams are integer multiples of the $d_{x}$ and $d_{y}$ factors given in Table 1, respectively.

Because the visibility is incompletely sampled and suffers from statistical fluctuations in practice, and because the direct transformation from visibility to brightness space may be ill-conditioned, regularization techniques and statistical inverse theory are applied to the reconstruction of the brightness distribution from the interferometry data. The particular algorithm used here employs entropy as the regularization metric [Ables, 1974; Jaynes, 1982; Skilling and Bryan, 1984]. A detailed description of the algorithm was given by Hysell and Woodman [1997].

\section{Radar images}

Images have been constructed from the $E$ region backscatter data in three dimensions; azimuth, zenith, and range. There being no expedient means of presenting three dimensional images in print, the zenith angle dependence of the images has been integrated out, although the moments of these integrals have been retained (see below). The resulting two-dimensional images are computed as functions of time and are essentially free of the spatial-temporal ambiguities inherent in RTI diagrams.

Figure 6 and Figure 7 show sequences of in-beam radar images of $E$ region field aligned irregularities. The horizontal and vertical axes of the image frames represent azimuth angle in degrees and range in kilometers, respectively. At a range of $170 \mathrm{~km}(260 \mathrm{~km})$, a $1^{\circ}$ angle corresponds to a transverse distance of about $3 \mathrm{~km}(4.5 \mathrm{~km})$. Note that the transmitting antennas mainly illuminate the central portions of the imaging fields of view, explaining why the image peripheries are frequently empty. Occasionally, however, strong echoes can be seen arriving from large azimuth angles through antenna sidelobes.

Interferometric cross-spectra were computed from the MU and Clemson radar data using 4- and 8-point fast Fourier transforms, respectively. Each image panel in Figure 6 and Figure 7 is really a composite of three separate images, computed from three of the available Doppler components of the cross-spectra. The three components were selected and color coded according to the key in Figure 8 and superimposed to form the composite images. Red, blue, and green colors denote red-shifted echo components, blue-shifted components, and components with small Doppler shifts. When combined this way, the lightness of each pixel becomes a representation of the signal-to-noise ratio (on a log scale, typically from 5-35 dB), the hue of the first moment Doppler velocity, and the saturation of the Doppler spectral width; pure (pastel) colors indicate narrow (broad) spectra.

Plotter symbols in the small graphs to the right of each 


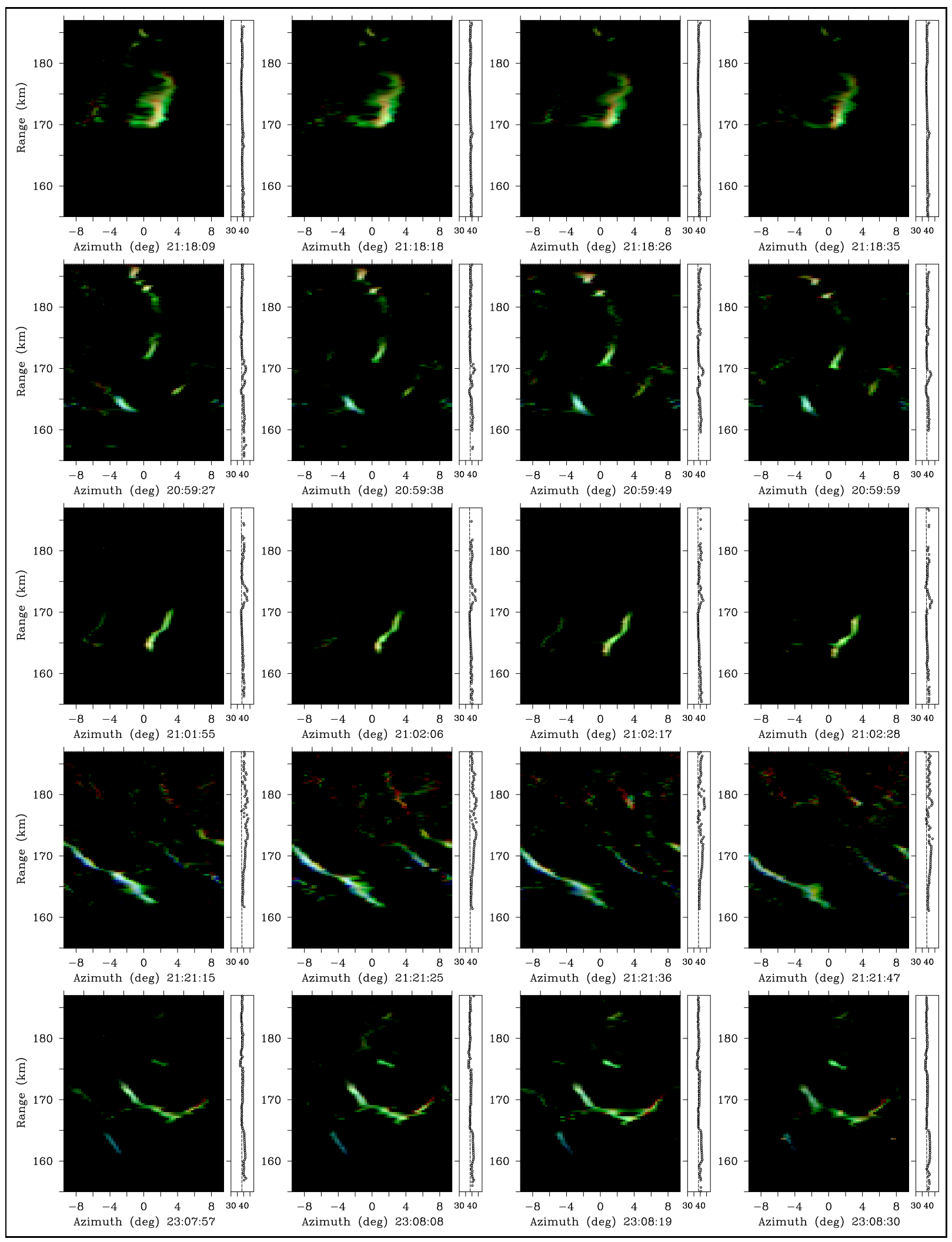

Figure 6. Sequences of four consecutive in-beam radar images computed from MU radar data. (top row) August 8,2001. (remaining rows) August 9, 2001. Times shown are LT. 


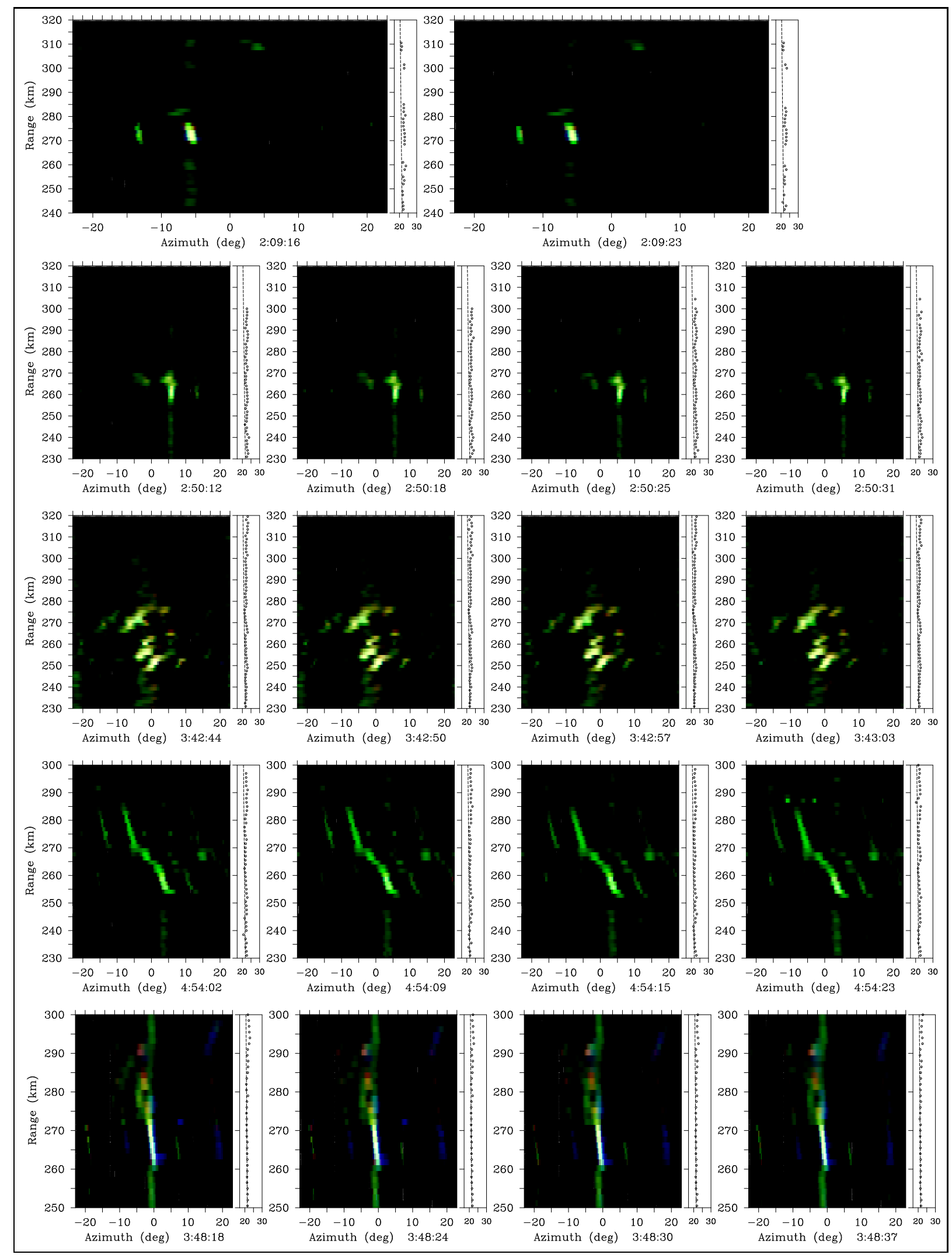

Figure 7. Sequences of two or four consecutive in-beam radar images computed from Clemson radar data. (top three rows) June 11, 2001. (fourth row) June 16, 2001. (bottom row) July 15, 2001. In the bottom row, images with red hues correspond to type I echoes. Times shown are UT. (UT=LT+5 hours). 


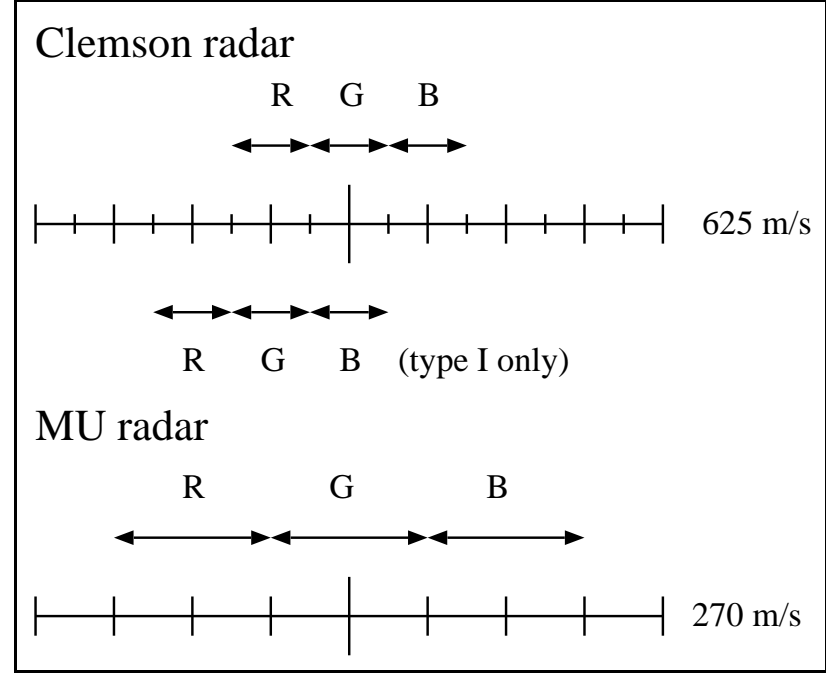

Figure 8. Correspondence between Doppler spectral bins and image color components for the MU and Clemson radar experiments. The respective Nyquist velocities for the experiments at both radars are given.

image panel in Figure 6 and Figure 7 show the elevation angle of the echo in degrees. The dashed lines drawn through these graphs mark the elevation angles anticipated for straight line propagation and perfectly field-aligned backscatter. Sometimes, the plotter symbols fall precisely along the dashed line. At other times, the measured elevation angles fall a few degrees off the dashed lines. Large discrepancies, thought to be mainly indicative of refraction, are more common in the Clemson than the MU radar data. The Clemson radar operates at a lower frequency and a lower elevation angle than the MU radar, and signals from it are more prone to experience the effects of refraction (see below).

\subsection{Type II echoes}

The images on the top row of Figure 6 typify the radar imaging dataset. In them, echoes are seen to arise from a localized region of space. The region is slightly elongated, being about $10 \mathrm{~km}$ deep in range but narrower in azimuth. Moreover, the Doppler shifts of the echoes are inhomogeneous and vary across the scattering region. The Doppler shifts are small at the edges of the scattering region but are distinctly red-shifted near the azimuthal center. These images suggest circulation within the scattering region, with electrons receding rapidly from the radar in the center but not at the periphery. Localized scattering regions with telltale signs of internal circulation are characteristic features of both radar datasets.

Over time, the scattering region depicted in the top row of Figure 6 drifted westward (toward the left of the image) while nearly maintaining its shape. In fact, all of the scatterers seen by the MU radar on August 8 drifted westward and had only very small meridional drift rates. That the echo signatures in the RTI plot in Figure 2 are amorphous rather than striated can be attributed to the predominately zonal motions of the underlying, localized scatterers which behave like point targets rather than volume scatter.

Note also that the backscatter in this case arrived from elevation angles close to what would be anticipated for perfectly field-aligned backscatter, as designated by the dashed lines in the small graphs to the right of the image panels. In fact, close inspection shows that the measured elevation angles fall just above the dashed line on the near side of the scattering region and just below it on the far side. The measured elevation angles imply that the backscatter in ranges between 170 and $180 \mathrm{~km}$ actually originated at a common altitude as if from a thin, horizontal, patchy layer. Examination of the entire imaging dataset reveals that it is typical for echoes from range-extended scattering regions to arrive from a single altitude and for that altitude to remain approximately constant as the scattering regions drift.

The second and third rows of images in Figure 6 show how multiple localized scattering regions can coexist within the radar illuminated volume. Though smaller, the scattering regions in these panels mainly share the characteristics of the one discussed above. The main difference is that these scatterers, observed on August 9, drifted rapidly toward the southwest. The numerous striations evident in Figure 1 between 2030 and 2200 LT are manifestations of multiple, sparsely packed scattering regions closing on the MU radar as they moved south westward through the illuminated volume. The closely spaced scattering regions at $180 \mathrm{~km}$ range and beyond in the second row of images in Figure 6, for example, were responsible for the "short period" QP echoes apparent just prior to 2100 in the RTI map.

Whereas all of the scattering regions discussed thusfar can be regarded as localized if elongated blobs, the fourth row of images in Figure 6 depicts a qualitatively different situation. Here, an extremely elongated ribbon of backscatter is evident. The ribbon extends from $0^{\circ}$ azimuth at 165 $\mathrm{km}$ range to the westward edge of the imaging field of view at $175 \mathrm{~km}$. Because of azimuth angle aliasing, the ribbon re-emerges on the eastward edge of the images and extends a few degrees further westward at greater and greater range. Over time, the ribbon can be seen to propagate southwestward in the direction perpendicular to the front. The scattering intensity along the ribbon is modulated, and each bright spot along it produced a striated signature in the RTI map in Figure 1. The Doppler characteristics of the ribbons are complicated but suggest red shifts in the body of the re- 
gions and blue-shifts or small Doppler shifts on the periphery.

Finally, more examples of localized scattering regions are shown in the bottom row of Figure 6. (Note: the echo between 160 and $165 \mathrm{~km}$ is a meteor echo and lies outside the bounds of this discussion.) These regions are elongated and do not share a common orientation. Over time, they drifted toward the southwest and, consequently, left striations in the RTI map in Figure 1.

The Clemson radar images shown in Figure 7 are substantially similar to the MU radar images, allowing for the inferior range resolution of the data from the former. The top two rows of Figure 7 show images corresponding to some of the striations apparent in Figure 3. Each of those striations arose from a localized, elongated, southward drifting scattering region. The irregularities indicated in the images here occupy a region about $10 \mathrm{~km}$ deep in range and about $1^{\circ}$ wide in azimuth. As with the MU radar images, the scattering regions shown here have inhomogeneous Doppler shifts and tend to be red shifted in the center and blue shifted at the edges.

The third row of Figure 7 presents images from later in the evening on June 11, 2001. By this time, the scattering regions had begun to drift predominantly westward, exhibiting only small meridional drifts of either sign. The numerous scattering regions evident in the images were responsible for the blob-like features in the corresponding RTI diagram. The fourth row of Figure 7, meanwhile, shows an example of a very long ribbon of backscatter seen by the Clemson radar. This ribbon extended about 30 and $70 \mathrm{~km}$ in the meridional and zonal directions, respectively, and was less than $10 \mathrm{~km}$ wide at any point. The Doppler shifts of the echoes were uniformly small in this case. Like the other, this ribbon propagated south westward over time in a direction normal to the front.

Animated sequences of images from all of the radar observations demonstrate that both the striations and the amorphous blobs in the radar RTI maps can be associated with localized, long-lived scattering regions drifting through the radar illuminated volume. Striations result when the regions drift in the direction of the radar. Negative and positive QP echo striation slopes correspond to southward and northward drifting scatterers, respectively. Most often, the scattering regions are observed drifting southwest before midnight. However, the field aligned nature of the scattering irregularities must impose boundaries on the scattering volume and limit the ranges from which echoes can be received. We can investigate these boundaries by examining the zenith angles of the QP echoes.

Figure 9 shows examples of Clemson radar data from June 11, 2001, and MU radar data from August 9, 2001, during periods when multiple quasiperiodic echoes were received. The echoes in question arrived from small azimuth angles. The echoes have been sorted by range and elevation angle and plotted in a grayscale format representing the relative signal-to-noise level on a dB scale. Each grouping of plotted pixels corresponds to echoes from an individual QP striation accumulated over time. The dashed lines in the figures predict the relationship between range and elevation angle for echoes originating at a fixed altitude. The solid lines represent the locus of perpendicularity. The evidence of the figure is that the scatterers in question are better ordered by altitude than by magnetic aspect angle.

In the case of the Clemson radar observations, the echoes only spanned one or two range gates at any given time, and their range followed curves of constant altitude as they approached the radar. In the case of the higher resolution MU radar data, the echoes also occupied several adjacent range gates in every integration time. Even so, all of the echoes associated with each given striation arrived from a common altitude. Evidently, the scattering regions responsible for the echoes are not only localized in range and azimuth but also in altitude, and they maintain altitude as they drift. Meanwhile, the elevation angles of the backscatter detected by the MU and Clemson radars can be seen here to depart from what is expected for field-aligned backscatter by as much as about $1.5^{\circ}$ and $2^{\circ}$, respectively. Similar observations were presented by Yamamoto et al. [1994].

Using radar interferometry to probe the equatorial electrojet at Jicamarca, Kudeki and Farley [1989] measured aspect angle half widths decreasing linearly with altitude from about $0.35^{\circ}$ at $98 \mathrm{~km}$ to about $0.1^{\circ}$ at $114 \mathrm{~km}$. Repeating their analysis on the MU radar data shown in Figure 9, we measure aspect angle half widths between about $0.25^{\circ}-1.0^{\circ}$. While the smallest of these angles are roughly consistent with the Jicamarca results for the altitudes indicated in Figure 9, the largest, which were measured in range gates containing multiple targets, reflect the fact that those targets reside at different altitudes. Moreover, as Figure 9 shows, the intensities of the echoes from the targets peak at elevation angles other than the presumptive loci of perpendicularity.

\subsection{Type I echoes}

Type I echoes are a relatively rare phenomenon at midlatitudes and were observed by the Clemson radar on a single occasion in 2001 on July 15, 2001 for an interval of about 2 minutes beginning at about 0348 UT in ranges between 280 and $295 \mathrm{~km}$. The spectra of the echoes were narrow and had Doppler shifts of about $250 \mathrm{~m} / \mathrm{s}$ with the sense of propagation away from the radar. Type II echoes were observed simultaneously but at closer range. Images of all the echoes are presented in the bottom row of Figure 7. These were 


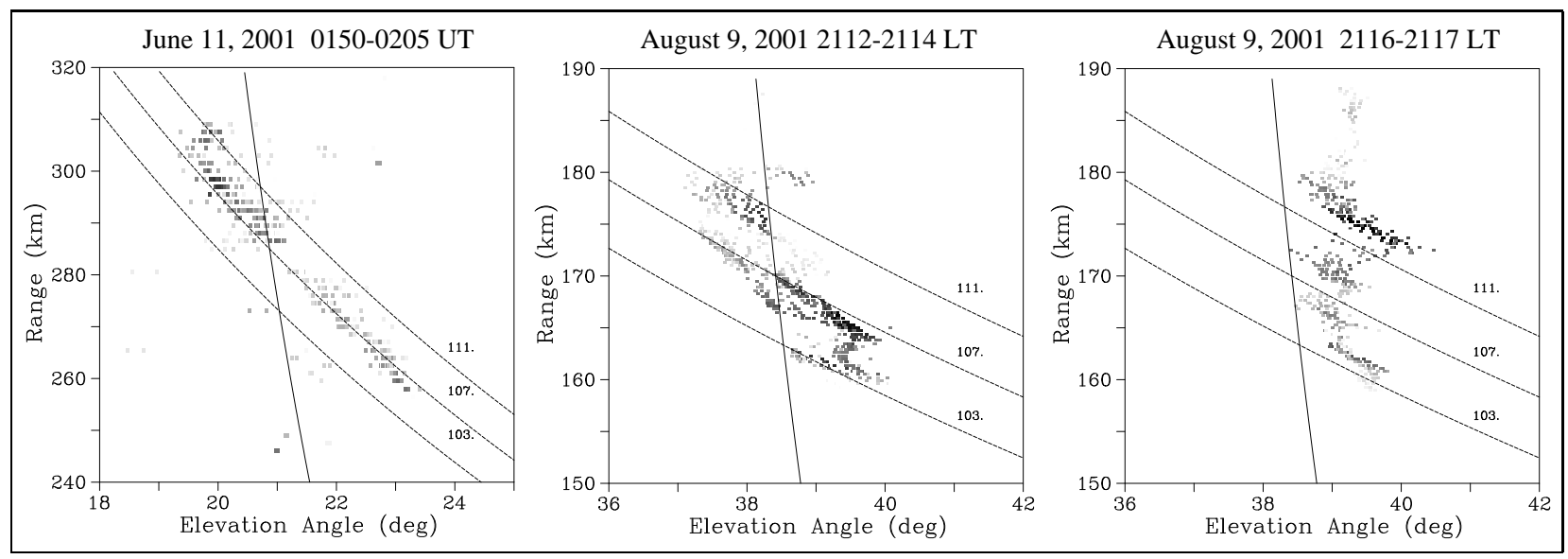

Figure 9. Comparison between echo ranges and elevation angles for groupings of scatterers. Grayscales indicate relative echo intensity on a dB scale. Horizontal curves trace the anticipated relationship between range and elevation angle for scatterers at the altitudes indicated. Vertical curves represent the locus of perpendicularity for field-aligned backscatter. (left panel) Clemson radar data. (center and right panels) MU radar data.

calculated in the same manner as the other images with one exception; the Doppler spectral bins utilized were shifted as shown in the key in Figure 8. Shifting the bins this way included the type I echoes in the red image component and also excluded some interference present at positive Doppler frequencies at the time in question.

The strong echoes in the images at ranges between about 260 and $275 \mathrm{~km}$ have type II spectra. The scattering region that gave rise to the echoes is very elongated and is tilted westward. The Doppler spectra of the echoes from the horizontal center of the region are broad and red shifted while the echoes from the periphery have narrow spectra with small Doppler shifts. Again, the elongated scattering region shows signs of internal circulation. The regions from where type I echoes were received, meanwhile, resolve as point targets. While they reside at higher altitudes, they fall along the line of the elongated, tilted scattering region. All of the echoes arrived from elevation angles close to the presumptive locus of perpendicularity. Note that the weak, green vertical lines at the center of the images are artifacts from the range sidelobes of the coded radar pulse and should be ignored.

\section{Analysis}

Conventional radar RTI analysis may not be sufficiently incisive to differentiate between competing theories of midlatitude $E$ region plasma irregularities. However, some of the ambiguity inherent in coherent scatter radar experiments can be mitigated with in-beam radar imaging. The processes responsible for QP echoes become clearer when radar images are interpreted in light of the theoretical framework outlined in paper 1 and of findings from other remote sensing and in situ experiments.

The electrodynamic consequences of ionization patches or clouds in the midlatitude $E$ region were investigated in paper 1 . Such patchy sporadic $E$ layers have been seen at Arecibo by Miller and Smith [1978] and Smith and Miller [1980] and have also been inferred from radio scintillations [Bowman, 1989; Maruyama, 1991]. In paper 1, we showed that the clouds can develop very large polarization electric fields if elongated and that strong currents can flow in the clouds an remain solenoidal by closing in the $F$ region. Drivers for the polarization include the background horizontal electric field imposed at night by the $F$ region dynamo along with horizontal $E$ region winds. Climatologically, the midlatitude meridional electric field is expected to be southward and have an amplitude of the order of $1 \mathrm{mV} / \mathrm{m}$ at night while the zonal electric field is expected to be significantly smaller [Richmond et al., 1980]. The horizontal winds in the lower thermosphere, meanwhile, exhibit considerable variability, as demonstrated recently by Larsen [2002]. The Horizontal Wind Model (HWM) indicates a broad tendency for the horizontal winds in the midlatitude $E$ region to be south westward in the early evening, shifting to north eastward by midnight [Hedin et al., 1996]. In the premidnight sector, therefore, we would expect $E$ region plasma clouds to drift south westward and to experience a predominantly southward or south eastward background electric field in their frame of reference, giving rise to a strong eastward or north eastward polarization electric field within. Polarization field strength of up to $10-20 \mathrm{mV} / \mathrm{m}$ are consistent with model results and have been detected in ground-based and in situ ex- 
periments [Pfaff et al., 1998; Haldoupis and Schlegel, 1994]. The conjunction of plasma density inhomogeneities, strong electric fields, and strong currents in plasma clouds suggests an association with plasma instabilities, plasma turbulence, small-scale plasma irregularities, and coherent scatter.

Because of the resemblance of midlatitude coherent scatter spectra to type II spectra observed in the equatorial electrojet, gradient drift instabilities are commonly assumed to be present in the sporadic $E$ layers. Rosado-Roman et al. [1999] showed how intermediate-scale (tens of meters) gradient drift modes with finite parallel wavenumber can be unstable in the midlatitude $E$ region. In paper 1, it was also shown that large-scale $(\sim 1 \mathrm{~km})$ collisional drift modes involving finite parallel wavenumbers can also be unstable in $E$ region plasma clouds. Evidence that the latter are found in nature was provided recently by Kelley et al. [1995] who conducted a rocket experiment in a volume in which coherent echoes were simultaneously detected by radar. The rocket payload detected clear signatures of horizontal, kilometric plasma density structuring in a plasma cloud situated above a sporadic $E$ layer in which intermediate-scale plasma waves were also embedded.

Whether the primary waves in question are kilometric collisional drift waves or intermediate-scale gradient drift waves, we can assume that small-scale plasma irregularities detected by radars arise from them through the turbulent processes described by Sudan [1983]. The phase velocity, $\omega / k$, for the small-scale waves is generally taken to be governed by

$$
\omega=\frac{\mathbf{k} \cdot\left(\mathbf{V}_{d e}-\mathbf{V}_{d i}\right)}{1+\psi}+\mathbf{k} \cdot \mathbf{V}_{d i}
$$

where $\psi$ is the anisotropy factor (the ratio of the electron to ion transverse mobility), $\omega$ is the frequency, $k$ is the wavenumber, and $\mathbf{V}_{d e}$ and $\mathbf{V}_{d i}$ are the electron and ion drift velocities, respectively. While this expression is strictly true for primary gradient drift and Farley Buneman waves, experience has shown that it describes the propagation of smallscale irregularities in the equatorial electrojet as well (see Fejer and Kelley [1980] for example). The electron drift velocity in (1) is approximately the $\mathbf{E} \times \mathbf{B}$ drift speed which reflects the effects if the background electric field, the polarization electric field that may arise in the plasma cloud, and the polarization electric field associated with the primary waves. The ion drift velocity, meanwhile, is mainly controlled by the neutral wind. The simulation studies in paper 1 indicate that, of these, the polarization electric field set up by the plasma cloud itself is likely to dominate at altitudes where the $\psi$ factor is small. The polarization field set up by primary waves may well become very strong under some circumstances, but its rapid variation in azimuth will generally be beyond the abilities of the radar to resolve. Neutral wind effects may dominate (1) at altitudes where $\psi$ is comparable to unity and below, but this domain is a source of only a small minority of the echoes we encountered. Most of the scatterers observed occupied altitudes above $100 \mathrm{~km}$ where $\psi$ is much less than unity.

The combination of a southward meridional electric field and a westward wind will set up polarization electric fields and electron circulation in $E$ region plasma clouds like those shown in Figure 3 of paper 1. There, the electron streamlines indicate rapid northward drifts in the center of the clouds and slower southward drifts on the cloud edges, in the rest frame of the cloud. In a stationary frame, the electron motion at the cloud edges could appear to be northward or southward depending on the meridional neutral wind speed. To the extent that small-scale waves are available as tracers throughout the body of the cloud, significantly red-shifted echoes should be observed from the center of the clouds in accordance with (1) whereas relatively small Doppler shifts should be observed at the cloud peripheries. Such characteristic Doppler shift patterns appear throughout our imaging data, and we therefore associate the elongated scattering regions with dimensions of a few kilometers in the images with the plasma clouds simulated in paper 1 .

It is important to recognize, however, that nothing guarantees that small-scale irregularities will be present uniformly throughout the plasma cloud to "paint" the entire circulation cell for the radar. Just as the clouds are distributed sparsely throughout the radar illuminated volume, the irregularities are distributed inhomogeneously throughout the cloud, and only part of the circulation is likely to be visible at any given time. Often, however, enough of the circulation is visible to make it recognizable.

\subsection{Refraction}

The finite aspect angle sensitivity of field aligned irregularities makes it possible for them to be observed for significant periods of time as they drift. Hysell and Burcham [2000] calculated that irregularities with an aspect angle half width of $0.5^{\circ}$ could be detected by the Clemson radar over a $50 \mathrm{~km}$ span of ranges, the length of the longest QP echo striation ever seen at Clemson. However, Figure 9 shows that the coherent echoes are not only spread in magnetic aspect angle beyond what is expected on the basis of experiments at Jicamarca, but also that they can be most intense at elevation angles well off the presumptive locus of perpendicularity. We attribute these phenomena mainly to refraction and seek to quantify the effects below.

We work in the domain of geometric optics and neglect birefringence, taking the radar frequency to be well above the critical frequency of the plasma. Consider the case of a 


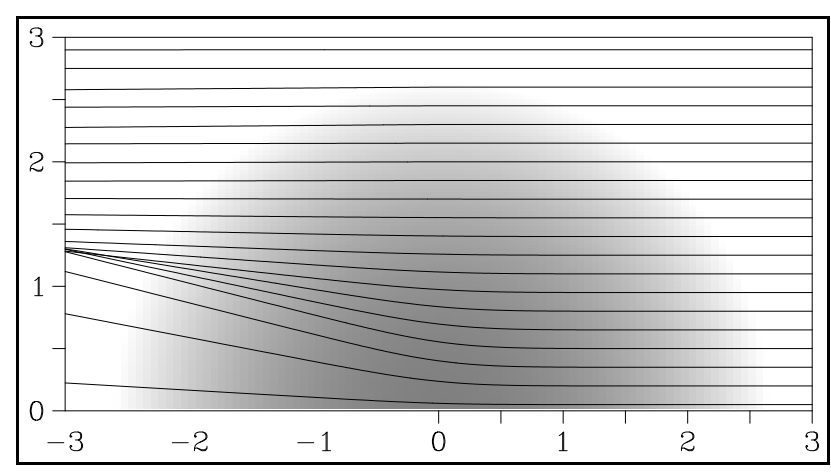

Figure 10. Paths of rays penetrating a plasma cloud with a Gaussian spheroid density distribution with $\sigma=0.5$. The axes represent nondimensional distances. The critical frequency is close to the radar frequency in this case, which is exaggerated for demonstration purposes.

spherically symmetric plasma cloud with density given by $N=N_{\circ} \exp \left(-r^{p} / 2 \sigma^{p}\right)$ where $r$ is the radial distance from the center. It is well known that, in the case of a medium with spherical symmetry, the ray characteristic in polar coordinates is governed by the ordinary differential equation

$$
\frac{d r}{d \theta}=\frac{r}{c} \sqrt{n^{2} r^{2}-c^{2}}
$$

where $n$ is the index of refraction and $c$ is an integration constant (e.g. Born and Wolf [1964]). We can integrate (2) numerically using the specified plasma cloud model and keep track of the characteristics of rays aimed at the cloud from a great distance. Integration is performed here using a predictor corrector method.

An example calculation is pictured in Figure 10. In this figure, we may regard the rays as having entered the region from the right in parallel as if from a distant source. When the rays exit the cloud on the left, they do so with a spread of trajectories. This spread may permit the Bragg scattering condition to be met at various places inside the cloud even if the incident rays are not orthogonal to the magnetic field. We have performed numerous calculations of this kind for a range of radar frequencies $f$, peak plasma densities $N_{\circ}$, and orders $p$. It was found that the maximum angular deflection of the rays from the horizontal $(\phi)$ in each case is predicted by the simple empirical law:

$$
\phi=2\left(\frac{30}{f}\right)^{2} \frac{N_{\circ}}{10^{6}}\left(\ln _{2} p+1\right)
$$

where the units of $\phi, f$, and $N_{\circ}$ are degrees, $\mathrm{MHz}$, and $\mathrm{cc}^{-1}$, respectively. This formula assumes that the radar frequency is much greater than the peak critical frequency and is accurate to within a few percent over a broad range of parameters.
Note that the problem is scale invariant such that $\sigma$ does not enter into (3). The maximum deviation angle $\phi$ provides an indication of the influence irregular, patchy sporadic $E$ layers are likely to have on the measured elevation angles of fieldaligned backscatter from irregularities inside the layers.

Ionograms taken throughout the evening of August 9, 2001, at the MU radar showed sporadic $E$ layers with top frequencies between 10 and $13 \mathrm{MHz}$. The blanking frequencies, meanwhile, were only about $2 \mathrm{MHz}$, supporting the notion that the layers were patchy or cloud like. Although MU radar and ionosonde do not probe a common volume, we may take the vertical soundings as an indication of the environment from which the quasiperiodic echoes emerged. According to (3), spherically symmetric plasma clouds with Gaussian distribution functions $(p=2)$ and with peak densities of just $1 \times 10^{6} \mathrm{~cm}^{-3}$ could give rise to deflections at $46.5 \mathrm{MHz}$ of up to $1.66^{\circ}$. This angle is close to the maximum departure from the locus of perpendicularity for the MU radar backscatter analyzed in Figure 9. Clouds with steeper boundaries could cause even greater deflection, and the deflections associated with Clemson radar signals should be correspondingly larger. With $N_{\circ}=4.5 \times 10^{5} \mathrm{~cm}^{-3}$ and $p$ $=2$, (3) predicts a maximum deflection of $1.8^{\circ}$ for Clemson radar signals, in close agreement with the maximum departures from perpendicularity apparent in Figure 9. Although a systematic study of the plasma distribution within patchy sporadic $E$ layers remains to be performed, plasma clouds with rather diffuse boundaries and with peak densities consistent with ionospheric soundings can account for the range of magnetic aspect angles observed in the coherent backscatter. This phenomenon allows the localized scattering regions underlying quasiperiodic echoes to be tracked by the radar as they drift over considerable distances.

Allowing that refraction can account for $\pm 1.5^{\circ}$ deviations in the radar ray paths and taking $0.25^{\circ}$ as a representative value of the aspect angle half widths of the field aligned irregularities, we can expect coherent echoes to be received from angles as far as $\pm 2.5^{\circ}$ from the locus of perpendicularity while suffering less than $35 \mathrm{~dB}$ of attenuation at the extremes. Striations associated with such echoes could have detectable range extents as great as about $20 \mathrm{~km}$ in typical MU radar RTI plots, the approximate length of the longest striations visible in Figure 1 and Figure 2. This figure becomes about $60 \mathrm{~km}$ for the Clemson radar. Echoes received from large azimuth angles could have considerably longer range extents than these, depending on the scattering geometry. Echoes received through sidelobes would have particularly large ranges and range extents. 


\subsection{Continuous bands (ribbons)}

In the MU and Clemson radar images, we found examples of extremely long bands or ribbons of backscatter. These represent the minority of the scatterers observed at both sites but were noted to occur several times in our dataset. Since the ribbons are modulated in intensity along their length, they are not easily distinguished in RTI maps from other, more common kinds of scatterers and leave striations as signatures. The ribbons have all been observed propagating toward the southwest.

A possible interpretation for the ribbons is that they represent discontinuities in ordinary, planar sporadic $E$ layers. In paper 1, it was found that ripple discontinuities in extended layers can become polarized in a background electric field, although the resulting polarization field is not necessarily very intense. How such a ripple would be induced in a sporadic $E$ layer is unknown. The morphology of the ribbons is reminiscent of the ripples in the total electron content observed by Saito et al. [1998, 2001] with the GSI network of global positioning satellite receivers in Japan (GEONET) during the passage of traveling ionospheric disturbances (TIDs). We may hypothesize that the ribbon scatterers reflect the $E$ region response to electrified TIDs propagating in the $F$ region across common magnetic field lines. A similar hypothesis was advanced recently in detail by Tsunoda and Cosgrove [2001]. In the future, radar images can be compared with images from the GEONET network to investigate commonalities in the two phenomena.

\subsection{Type I echoes}

The dispersion of Farley Buneman waves, of which type I echoes are a signature, is governed by (1) along with the following growth rate expression:

$$
\gamma=\frac{\psi / \nu_{i}}{1+\psi}\left(\left(\omega-\mathbf{k} \cdot \mathbf{V}_{d i}\right)^{2}-k^{2} C_{s}^{2}\right)-2 \alpha n_{\circ}
$$

where $\nu_{i}$ is the ion-neutral collision frequency, $C_{s}$ is the ion acoustic speed, and $\alpha$ is the recombination rate (e.g. Fejer et al. [1975].) When combined with (1), (4) implies a threshold velocity for the electrons below which waves cannot be excited. Neglecting recombination, the threshold condition is

$$
v_{d e}>v_{d i}+C_{s}(1+\psi)
$$

where the scalar drifts refer to the component of the electron and ions in the direction of wave propagation. Favorable conditions for the generation of Farley Buneman waves include low temperatures and metallic ion composition, which reduce the ion acoustic speed, along with winds and ion drifts in the direction opposite the electron drifts. Finally, the anisotropy factor is much less than unity above about $100 \mathrm{~km}$ but increases rapidly below, drastically increasing the electron drift threshold there and making Farley Buneman waves more difficult to excite.

The images in the bottom row of Figure 7 depict a highly elongated scattering region between about 260-270 $\mathrm{km}$ range showing all the signs of being strongly polarized. The elevation angles of this type II echo region are close to the locus of perpendicularity, suggesting minimal refraction, but are larger at the near edge than at the far edge, implying that the elongated scattering region is a horizontal layer. At ranges beyond $270 \mathrm{~km}$, there is a region devoid of backscatter and then two compact regions with type I echoes that fall along the line of the elongated scattering region. The altitudes of the type II and type I echo regions are about 100 and $110 \mathrm{~km}$, respectively.

That all the scattering regions in the radar images are collinear suggests that they are related even though the type I and II echo regions are separated by $10 \mathrm{~km}$ in altitude. We may surmise that the elongated plasma layer responsible for the type II echoes extended to ranges greater than $270 \mathrm{~km}$ but that the Bragg scattering condition was unmet beyond $270 \mathrm{~km}$, and so no echoes were received. Assuming that strong polarization electric fields were present in the layer at more distant ranges, these fields would have mapped along magnetic field lines to higher altitudes at which the Bragg scattering condition was once again met. At $110 \mathrm{~km}$ altitude, the mapped polarization electric field could have excited Farley Buneman instabilities and given rise to the type I echoes, provided the threshold condition was met at the higher altitude. Why type I echoes appeared at the higher altitude but not the lower in that case is unclear. Vertical gradients in the winds, composition, and the $\psi$ factor presumably reduced the instability threshold in the upper layer to the point where instabilities could be excited.

\section{Summary and Conclusions}

We have investigated the scattering regions underlying QP echoes using in-beam radar imaging. These regions turn out to be spatially localized, to be horizontally elongated, and to maintain altitude as they drift through the radar illuminated volume. The trademark quasiperiodic striations in radar RTI plots are the trails of the drifting scatterers, like the trails of stars left in long-duration photographic exposures. Refraction can permit coherent scatter from field aligned irregularities within the regions to be detected over a wide range of elevation angles. The climatology of the dynamo electric field and the neutral winds in the midlatitude lower thermosphere is consistent with the most commonly observed signs of the echo range rates and Doppler shifts. 
Plasmas are charge neutral by definition, and the assemblage of free charge in a plasma is energetically expensive. However, a dielectric plasma response leading to the creation of induced electric dipoles is energetically favorable in a plasma in an applied field and does not violate charge neutrality. Effective charge appears wherever the polarization (induced dipole moment per unit volume) is divergent and tends to concentrate near the boundaries or surfaces of inhomogeneous plasma structures. The polarization electric fields that arise are dipolar or higher order. Electrons, with streamlines that follow the equipotentials, will circulate in a conspicuous way when drifting through inhomogeneous plasmas.

Circulation has been uncovered using radar imaging. We interpret the circulation as evidence that the scattering regions coincide with elongated, polarized plasma clouds. Such clouds have been observed in the ionosphere over Arecibo and have been detected in other in situ and remote sensing experiments. Simulations show that polarization electric fields and Hall drifts large enough to drive Farley Buneman instabilities and produce type I echoes can arise in particularly elongated clouds coupled electrically to the $F$ region. The type I echoes detected by the Clemson radar occurred in the vicinity of a highly elongated scattering region. Simulations also show that plasma clouds are unstable to kilometer-scale collisional drift instability modes, and we presume at this point that intermediate- and smallscale irregularities can be generated subsequently by nonlinear mode coupling and plasma turbulence. Evidence of horizontal, kilometric waves in patchy sporadic $E$ layers that were accompanied by intermediate- and small-scale waves was presented by Kelley et al. [1995].

In paper 1 , it was argued that $E$ region plasma clouds produce all the phenomena necessary to account for smallscale irregularities and coherent radar backscatter including large-scale primary waves, large electric fields, and large Hall currents. Nevertheless, simultaneous observations of $E$ region plasma clouds, of structure within those clouds, and of QP echoes from a common volume remain unmade. Likewise, although there is compelling evidence that neutral shear instabilities may be responsible for producing patchy sporadic $E$ layers and clouds, direct observations of the process at work are lacking. Before a complete picture of the QP echo phenomenon can develop, simultaneous measurements of the background plasma density and electric fields (via incoherent scatter or rocket probes), intermediate-scale plasma structure (via rocket probes or radio scintillations), neutral wind profile (via incoherent scatter or chemical releases), and coherent scatter (via interferometry or imaging) will have to be made.
Acknowledgments. This work was supported by the National Science Foundation through NSF grant ATM-0080338 to Clemson University. Special thanks are due M. F. Larsen for helping with the fielding and operation of the Clemson radar. The MU radar belongs to and is operated by the Radio Science Center for Space and Atmosphere (RASC) of Kyoto University. DLH was supported by RASC as a visiting associate professor during the preparation of this manuscript.

\section{References}

Ables, J. G., Maximum entropy spectral analaysis, Astron. Astrophys. Suppl. Ser., 15, 383, 1974.

Born, M., and E. Wolf, Principles of Optics, Pergamon, New York, 1964.

Bowman, G. G., Quasi-periodic scintillations at mid-latitudes and their possible association with ionospheric sporadic- $E$ structures, Ann. Geophys., 7, 259, 1989.

Farley, D. T., H. M. Ierkic, and B. G. Fejer, Radar interferometry: A new technique for studying plasma turbulence in the ionosphere, J. Geophys. Res., 86, 1467, 1981.

Fejer, B. G., and M. C. Kelley, Ionospheric irregularities, Rev. Geophys., 18, 401, 1980.

Fejer, B. G., D. T. Farley, B. B. Balsley, and R. F. Woodman, Vertical structure of the VHF backscattering region in the equatorial electrojet and the gradient drift instability, J. Geophys. Res., 80, 1313, 1975.

Haldoupis, C., and K. Schlegel, Observation of the modified twostream plasma instability in the midlatitude $E$ region ionosphere, J. Geophys. Res., 99, 6219, 1994.

Haldoupis, C., K. Schlegel, and D. T. Farley, An explanation for type 1 radar echoes from the midlatitude $E$-region ionosphere, Geophys. Res. Lett., 23, 97, 1996.

Hedin, A. E., et al., Empirical wind model for the upper, middle, and lower atmosphere, J. Atmos. Terr. Phys., 58, 1421, 1996.

Hysell, D. L., and J. Burcham, The $30 \mathrm{MHz}$ radar interferometer studies of midlatitude $E$ region irregularities, J. Geophys. Res., 105, 12,797, 2000.

Hysell, D. L., and R. F. Woodman, Imaging coherent backscatter radar observations of topside equatorial spread $F$, Radio Sci., 32, 2309, 1997.

Hysell, D. L., J. L. Chau, and C. G. Fesen, Effects of large horizontal winds on the equatorial electrojet, J. Geophys. Res., 2002, in press.

Jaynes, E. T., On the rationale of maximum-entropy methods, Proc. IEEE, 70, 939, 1982.

Kagan, L. M., and M. C. Kelley, A wind-driven gradient drift mechanism for midlatitude $E$-region ionospheric irregularities, Geophys. Res. Lett., 25, 4141, 1998.

Kagan, L. M., and M. C. Kelley, A thermal mechanism for generation of small-scale irregularities in the ionospheric $E$-region, $J$. Geophys. Res., 105, 5291, 2000.

Kelley, M. C., D. Riggin, R. F. Pfaff, W. E. Swartz, J. F. Providakes, and C. S. Huang, Large amplitude quasi-periodic fluctuations associated with a midlatitude sporadic $E$ layer, J. Atmos. Terr. Phys., 57, 1165, 1995. 
Kudeki, E., and D. T. Farley, Aspect sensitivity of equatorial electrojet irregularities and theoretical implications, J. Geophys. Res., 94, 426, 1989.

Kudeki, E., and F. Sürücü, Radar interferometric imaging of fieldaligned plasma irregularities in the equatorial electrojet, Geophys. Res. Lett., 18, 41, 1991.

Larsen, M. F., A shear instability seeding mechanism for quasiperiodic radar echoes, J. Geophys. Res., 105, 24,931, 2000.

Larsen, M. F., Winds and shears in the mesosphere and lower thermosphere: results from four decades of chemical release wind measurements, J. Geophys. Res., 2002, in press.

Maruyama, T., Observations of quasi-periodic scintillations and their possible relation to the dynamics of Es plasma blobs, Radio Sci., 26, 691, 1991.

Maruyama, T., S. Fukao, and M. Yamamoto, A possible mechanism for echo-striation generation of radar backscatter from midlatitude sporadic E, Radio Sci., 35, 1155, 2000.

Miller, K. L., and L. G. Smith, Incoherent scatter radar observations of irregular structure in mid-latitude sporadic $E$ layers, $J$. Geophys. Res., 83, 3761, 1978.

Pfaff, R. F., M. Yamamoto, P. Marionnia, H. Mori, and S. Fukao, Electric field measurements above and within a sporadic- $E$ layer, Geophys. Res. Lett., 25, 1769, 1998.

Richmond, A. D., et al., An empirical model of quiet-day ionospheric electric fields at middle and low latitudes, J. Geophys. Res., 85, 4658, 1980.

Rosado-Roman, J. M., W. E. Swartz, and D. T. Farley, Radar studies in Puerto Rico of mid-latitude $E$-region plasma instabilities, paper presented at the XXVI URSI General Assembly, Toronto, Canada, August 13-21, 1999.

Saito, A., S. Fukao, and S. Miyazaki, High resolution mapping of TEC perturbations with the GSI GPS netwark over Japan, Geophys. Res. Lett., 25, 3079, 1998.

Saito, A., et al., Traveling ionospheric disturbances detected in the FRONT campaign, Geophys. Res. Lett., 28, 689, 2001.

Shalimov, S., C. Haldoupis, and K. Schlegel, Large polarization electric fields associated with midlatitude sporadic E, J. Geophys. Res., 103, 11,617, 1998.

Skilling, J., and R. K. Bryan, Maximum entropy image reconstruction: General algorithm, Mon. Not. R. Astron. Soc., 211, 111, 1984.

Smith, L. G., and K. L. Miller, Sporadic- layers and unstable wind shears, J. Atmos. Terr. Phys., 42, 45, 1980.

Sudan, R. N., Unified theory of type I and type II irregularities in the equatorial electrojet, J. Geophys. Res., 88, 4853, 1983.

Thompson, A. R., Interferometry and Synthesis in Radio Astronomy, John Wiley, New York, 1986.

Tsunoda, R. T., On polarized frontal structures, type-1 and quasiperiodic echoes in midlatitude sporadic E, Geophys. Res. Lett., 25, 2641, 1998.

Tsunoda, R. T., and R. B. Cosgrove, Coupled electrodynamics in the nighttime midlatitude ionosphere, Geophys. Res. Lett., 2001, in press.

Tsunoda, R. T., S. Fukao, and M. Yamamoto, On the origin of quasi-periodic backscatter from sporadic E, Radio Sci., 29, 349, 1994.

Woodman, R. F., Coherent radar imaging: Signal processing and statistical properties, Radio Sci., 32, 2373, 1997.
Woodman, R. F., M. Yamamoto, and S. Fukao, Gravity wave modulation of gradient drift instabilities in mid-latitude sporadic $E$ irregularities, Geophys. Res. Lett., 18, 1197, 1991.

Yamamoto, M., S. Fukao, R. F. Woodman, T. Ogawa, T. Tsuda, and K. Kato, Mid-latitude $E$ region field-aligned irregularities observed with the MU radar, J. Geophys. Res., 96, 15,943, 1991.

Yamamoto, M., S. Fukao, T. Ogawa, T. Tsuda, and S. Kato, A morphological study of mid-latitude $E$-region field-aligned irregularities observed with the MU radar, J. Atmos. Terr. Phys., 54, 769, 1992.

Yamamoto, M., N. Komoda, S. Fukao, R. T. Tsunoda, and T. Ogawa, Spatial structure of the $E$ region field-aligned irregularities revealed by the MU radar, Radio Sci., 29, 337, 1994.

Received X; revised X; accepted X.

This preprint was prepared with AGU's LTTE $_{\mathrm{E}} \mathrm{X}$ macros v5.01, with the extension package 'AGU'+', by P. W. Daly, version 1.6b from 1999/08/19. 